\title{
Generalized Poisson Summation Formulas for Continuous Functions of Polynomial Growth
}

\author{
Ha Q. Nguyen ${ }^{1}$ - Michael Unser ${ }^{1}$. \\ John Paul Ward ${ }^{1,2}$
}

Received: 28 July 2015 / Published online: 24 May 2016

(C) Springer Science+Business Media New York 2016

\begin{abstract}
The Poisson summation formula (PSF) describes the equivalence between the sampling of an analog signal and the periodization of its frequency spectrum. In engineering textbooks, the PSF is usually stated formally without explicit conditions on the signal for the formula to hold. By contrast, in the mathematics literature, the PSF is commonly stated and proven in the pointwise sense for various types of $L_{1}$ signals. This $L_{1}$ assumption is, however, too restrictive for many signal-processing tasks that demand the sampling of possibly growing signals. In this paper, we present two generalized versions of the PSF for $d$-dimensional signals of polynomial growth. In the first generalization, we show that the PSF holds in the space of tempered distributions for every continuous and polynomially growing signal. In the second generalization, the PSF holds in a particular negative-order Sobolev space if we further require that $d / 2+\varepsilon$ derivatives of the signal are bounded by some polynomial in the $L_{2}$ sense.
\end{abstract}

Communicated by Eric Todd Quinto.

This work was presented in part at the International Conference on Sampling Theory and Applications, Washington, DC, USA, May 2015.

$凶$ Ha Q. Nguyen

ha.nguyen@epfl.ch

Michael Unser

michael.unser@epfl.ch

John Paul Ward

john.ward@ucf.edu

1 Biomedical Imaging Group, École Polytechnique Fédérale de Lausanne (EPFL), 1015 Lausanne, Switzerland

2 Present Address: University of Central Florida, Orlando, FL 32816, USA 
Keywords Poisson summation formula $\cdot$ Sampling theory $\cdot$ Polynomially growing functions · Weighted Sobolev spaces · Tempered distributions

\section{Introduction}

A widely used result in signal processing is that sampling in the space domain corresponds to periodization in the frequency domain and vice versa. This relation is mathematically described by the Poisson summation formula

$$
\sum_{\boldsymbol{k} \in \mathbb{Z}^{d}} f(\boldsymbol{k}) \mathrm{e}^{-2 \pi \mathrm{j}(\boldsymbol{k} \cdot)}=\sum_{\ell \in \mathbb{Z}^{d}} \hat{f}(\cdot+\ell),
$$

where the left-hand side (LHS) is the discrete-space Fourier transform of the discretized signal $\{f(\boldsymbol{k})\}_{\boldsymbol{k} \in \mathbb{Z}^{d}}$, and the right-hand side (RHS) is the periodization of the continuous-space Fourier transform of the analog signal $\{f(\boldsymbol{x})\}_{\boldsymbol{x} \in \mathbb{R}^{d}}$. The PSF lies at the heart of sampling theories including the classical Shannon's sampling theorem [25] because it helps connect the digital world to the analog world through the Fourier transform.

In the mathematics literature, the PSF is often stated the other way round with the periodization appearing in the space domain and the sampling occurring in the Fourier domain. Throughout this paper, however, we shall consistently refer to Equation (PSF) as the statement of the PSF. Various versions of the PSF have been proved in the pointwise sense when both the signal $f$ and its Fourier transform $\hat{f}$ are in appropriate subspaces of $L_{1}\left(\mathbb{R}^{d}\right) \cap \mathcal{C}\left(\mathbb{R}^{d}\right)$, where $\mathcal{C}\left(\mathbb{R}^{d}\right)$ is the space of continuous functions. Given that $f, \hat{f} \in L_{1}\left(\mathbb{R}^{d}\right) \cap \mathcal{C}\left(\mathbb{R}^{d}\right)$, the LHS of PSF can be interpreted as the (possibly divergent) Fourier series of the periodic function in the RHS. Note that, by Katznelson's counterexample [15], it is not sufficient to require $f, \hat{f}$ to be continuous and in $L_{1}$. One way to make PSF valid pointwise is to impose an appropriate decay on both the signal and its Fourier transform. For example, if $f$ and $\hat{f}$ are continuous and satisfy the decay

$$
|f(\boldsymbol{x})|+|\hat{f}(\boldsymbol{x})| \leq C(1+\|\boldsymbol{x}\|)^{-d-\varepsilon}, \quad \forall \boldsymbol{x} \in \mathbb{R}^{d},
$$

for some $C, \varepsilon>0$, then it was shown [10,27] that PSF holds pointwise with absolute convergence of both sides. More generally, Kahane and Lemarié-Rieusset [14] considered signals $f$ such that $\|\boldsymbol{x}\|^{a} f \in L_{p}\left(\mathbb{R}^{d}\right)$ and $\|\xi\|^{b} \hat{f} \in L_{q}\left(\mathbb{R}^{d}\right)$, with $a, b \geq 0$, and gave a characterization of parameters $a, b, p, q$ such that the PSF holds. Gröchenig obtained in [11] a similar result in connection with the uncertainty principle. In the 1 -D case, when $d=1$, it is also known [4,36] that PSF holds pointwise when $f, \hat{f} \in L_{1}(\mathbb{R}) \cap \mathcal{C}(\mathbb{R})$ and $\hat{f}$ has bounded total variation. Many other forms of the PSF on $L_{1}(\mathbb{R})$ can also be found in the extensive work of Benedetto and Zimmermann [4]. The main limitation of all the above versions of the PSF is that they do not apply to functions that might be growing, such as realizations of a non-stationary stochastic process, the typical example being Brownian motion [31]. Surprisingly, the 
existing forms of the PSF cannot even handle the simple (1-D) one-sided power signals $x_{+}^{\alpha}:=(\max (0, x))^{\alpha}$, for $\alpha>0$.

What appears to be missing is a rigorous proof of the PSF for a broad class of signals beyond the $L_{1}$ hypothesis. In engineering texts, see for example [6,20,21], the PSF is often stated without explicitly specifying sufficient conditions on the signal $f$. The common proof of the PSF that is presented to engineers is based on symbolic manipulations with the following steps: (1) The LHS of PSF is the Fourier transform of the multiplication between $f$ and the Dirac comb $\delta_{\text {period }}:=\sum_{\boldsymbol{k} \in \mathbb{Z}^{d}} \delta(\cdot+\boldsymbol{k})$; (2) the RHS of PSF is the convolution of $\hat{f}$ with $\delta_{\text {period; }}$ and (3) the two sides are equal due to the convolution theorem and to the fact that $\delta_{\text {period }}$ is the Fourier transform of itself. The problem with this argument, in light of Schwartz' distribution theory [24], is that the multiplication of a Dirac comb with an arbitrary function is not necessarily a tempered distribution, so that the convolution theorem may not be applicable. This can also result in erroneous conclusions such as the distributional version of Shannon's sampling theorem in [34, Theorem7.6.1]. In fact, various generalizations of the sampling theorem for bandlimited functions of polynomial growth were rigorously established in [7,12,16,22,32,33,35]. More recently, Fischer [8] fixed the Dirac-comb-based argument in proving the PSF by requiring the signal $f$ to be infinitely differentiable and bounded by a polynomial. This condition, although allowing the signal to grow, is unnecessarily restrictive. Intuitively, in order for the signal to be sampled, it only needs to be continuous.

Our work is an attempt to bridge the gap between the engineering and mathematics statements of the PSF. We provide in this paper two generalizations of the PSF for (possibly) growing signals. In the first generalization, we prove that PSF holds in the space $\mathcal{S}^{\prime}\left(\mathbb{R}^{d}\right)$ of tempered distributions if the signal $f$ is continuous and bounded by a polynomial. This condition is minimal for the signal to be sampled and for its Fourier transform to make sense. In this case, the RHS of PSF should be interpreted as a Cesàro sum which converges conditionally, and the two sides are identical continuous linear functionals on Schwartz' space $\mathcal{S}\left(\mathbb{R}^{d}\right)$ of infinitely differentiable and rapidly decaying functions. The second generalization of the PSF is for signals whose $d / 2+\varepsilon$ derivatives, for arbitrary $\varepsilon>0$, are bounded by a polynomial in the $L_{2}$ sense. In particular, if $f$ is included in the weighted Sobolev space $L_{2,-2 n}^{d / 2+\varepsilon}\left(\mathbb{R}^{d}\right)$ with order of differentiability $d / 2+\varepsilon$ and with order of growth $2 n$, for $n \in \mathbb{N}$, then PSF holds in the corresponding negative-order Sobolev space $L_{2}^{-2 n}\left(\mathbb{R}^{d}\right)$. In this version, both sums in PSF converge unconditionally; hence no need for summability methods. Moreover, it should be understood that the two sides of PSF are equal in $L_{2}^{-2 n}\left(\mathbb{R}^{d}\right)$ in the sense that their Fourier transforms are growing functions that are equal almost everywhere. Alternatively, by duality, the second generalized PSF can be interpreted as the equality of two continuous linear functionals on the positive-order Sobolev space $L_{2}^{2 n}\left(\mathbb{R}^{d}\right)$.

This paper is a development of the previous works [18,19] of the first two authors. A weaker version of the generalized PSF on weighted Sobolev spaces was briefly introduced in [19] without detailed proofs. A general theory for sampling growing signals was given in [18]. The outline of the rest of the paper is as follows: In Sect. 2, we provide some mathematical preliminaries, mainly for the benefit of readers with 
an engineering background. In Sect. 3, we state and prove the first generalization of the PSF for polynomially growing functions. The second generalization of the PSF for functions in weighted Sobolev spaces is presented in Sect. 4. Finally, we provide a discussion in Sect. 5.

\section{Preliminaries}

\subsection{Notation}

Let $d \geq 1$ be a fixed dimension. All functions in this paper are complex-valued and multidimensional with variables in $\mathbb{R}^{d}$. Let $\|\boldsymbol{x}\|$ denote the Euclidean norm of a vector $\boldsymbol{x} \in \mathbb{R}^{d}$. For $\boldsymbol{k} \in \mathbb{N}^{d}$, we use the following standard multi-index notation:

$$
|\boldsymbol{k}|:=\sum_{i=1}^{d} k_{i}, \quad \boldsymbol{k} !:=k_{1} ! \cdots k_{d} !, \quad \boldsymbol{x}^{k}:=\prod_{i=1}^{d} x_{i}^{k_{i}}, \quad \partial^{k} f:=\frac{\partial^{|\boldsymbol{k}|}}{\partial \boldsymbol{x}_{1}^{k_{1}} \ldots \partial \boldsymbol{x}_{d}^{k_{d}}} f
$$

The constants throughout the paper are denoted by $C$ with subscripts denoting the dependence of the constants on some parameters. We denote by $f[\cdot]$ the sampled sequence of a function $f$ on the integer multigrid; that means $f[\boldsymbol{k}]:=f(\boldsymbol{k}), \forall \boldsymbol{k} \in \mathbb{Z}^{d}$. For $1 \leq p \leq \infty, L_{p}\left(\mathbb{R}^{d}\right)$ and $\ell_{p}\left(\mathbb{Z}^{d}\right)$ denote the spaces of functions and sequences, respectively, whose $p$-norms are finite. The scalar product of two functions $f$ and $g$ is defined by

$$
\langle f, g\rangle_{\mathbb{R}^{d}}:=\int_{\mathbb{R}^{d}} f(\boldsymbol{x}) g(\boldsymbol{x}) \mathrm{d} \boldsymbol{x},
$$

whenever the integral exists. Complex conjugation is unconventionally dropped in the definition of a scalar product to preserve bilinearity. For a topological vector space $V$ on the complex field $\mathbb{C}$, its dual space $V^{\prime}$ is defined to be the set of all continuous linear functionals $f: V \rightarrow \mathbb{C}$. The action of a functional $f \in V^{\prime}$ on a vector $\varphi \in V$ is denoted by $\langle f, \varphi\rangle$ (without the subscript $\mathbb{R}^{d}$ ). Background on topological vector spaces can be found in, for example, [23].

The forward and inverse Fourier transforms of a function $f \in L_{1}\left(\mathbb{R}^{d}\right)$ are defined by

$$
\begin{aligned}
\{\mathcal{F} f\}(\boldsymbol{\xi}):=\hat{f}(\boldsymbol{\xi}) & :=\int_{\mathbb{R}^{d}} f(\boldsymbol{x}) \mathrm{e}^{-2 \pi \mathrm{j}\langle\boldsymbol{\xi}, \boldsymbol{x}\rangle} \mathrm{d} \boldsymbol{x}, \\
\left\{\mathcal{F}^{-1} f\right\}(\boldsymbol{x}) & :=\int_{\mathbb{R}^{d}} f(\boldsymbol{\xi}) \mathrm{e}^{2 \pi \mathrm{j}\langle\boldsymbol{\xi}, \boldsymbol{x}\rangle} \mathrm{d} \boldsymbol{\xi},
\end{aligned}
$$

respectively, where $\mathrm{j}^{2}=-1$ and $\langle\boldsymbol{\xi}, \boldsymbol{x}\rangle:=\sum_{i=1}^{d} \xi_{i} x_{i}$. Note that the Fourier transform is not an isomorphism on $L_{1}\left(\mathbb{R}^{d}\right)$. However, by Plancherel's theorem and by the density of $L_{1}\left(\mathbb{R}^{d}\right) \cap L_{2}\left(\mathbb{R}^{d}\right)$ in $L_{2}\left(\mathbb{R}^{d}\right)$, the Fourier transform can be extended to an isometry 
on $L_{2}\left(\mathbb{R}^{d}\right)$, leading to

$$
\|\hat{f}\|_{L_{2}\left(\mathbb{R}^{d}\right)}=\|f\|_{L_{2}\left(\mathbb{R}^{d}\right)}, \quad \forall f \in L_{2}\left(\mathbb{R}^{d}\right) .
$$

More generally, for $f, g \in L_{2}\left(\mathbb{R}^{d}\right)$, Parseval's relation takes the form

$$
\langle f, g\rangle_{\mathbb{R}^{d}}=\left\langle\mathcal{F} f, \mathcal{F}^{-1} g\right\rangle_{\mathbb{R}^{d}}
$$

Note that, this form of Parseval's relation is unconventional ${ }^{1}$ due to the definition of scalar product in (1). The extension of the Fourier transform to the class of generalized functions called tempered distributions will be discussed in Sect. 2.3 with a brief review of the underlying theory.

A function $f$ is called polynomially growing (or of polynomial growth) if there exist $C>0$ and $n \in \mathbb{N}$ such that

$$
|f(\boldsymbol{x})| \leq C(1+\|\boldsymbol{x}\|)^{n}, \quad \forall \boldsymbol{x} \in \mathbb{R}^{d}
$$

Despite its name, a polynomially growing function is not necessarily growing but instead bounded poinwise by a polynomial. When we want to control the $L_{2}$-norm of a growing or decaying function, it is necessary to consider weighted- $L_{2}$ spaces. In particular, we define

$$
L_{2, n}\left(\mathbb{R}^{d}\right):=\left\{f: \int_{\mathbb{R}^{d}}|f(\boldsymbol{x})|^{2}\left(1+\|\boldsymbol{x}\|^{2}\right)^{n} \mathrm{~d} \boldsymbol{x}<\infty\right\},
$$

where the parameter $n \in \mathbb{Z}$ specifies the order of growth (when $n \leq 0$ ) or decay (when $n>0)$ of the function. The weighted norm of a function $f \in L_{2, n}\left(\mathbb{R}^{d}\right)$ is given by

$$
\|f\|_{L_{2, n}\left(\mathbb{R}^{d}\right)}:=\left(\int_{\mathbb{R}^{d}}|f(\boldsymbol{x})|^{2}\left(1+\|\boldsymbol{x}\|^{2}\right)^{n} \mathrm{~d} \boldsymbol{x}\right)^{\frac{1}{2}} .
$$

Given an $n \in \mathbb{N}$, all functions in $L_{2,-n}\left(\mathbb{R}^{d}\right)$ are said to be bounded by a polynomial of degree $n$ in the $L_{2}$ sense. It is not difficult to see that every function of polynomial growth must belong to $L_{2,-n}\left(\mathbb{R}^{d}\right)$ for some $n \in \mathbb{N}$.

Similarly, for $n \in \mathbb{Z}$, the space of weighted- $\ell_{2}$ sequences is defined by

$$
\ell_{2, n}\left(\mathbb{Z}^{d}\right):=\left\{c: \sum_{\boldsymbol{k} \in \mathbb{Z}^{d}}|c[\boldsymbol{k}]|^{2}\left(1+\|\boldsymbol{k}\|^{2}\right)^{n}<\infty\right\} .
$$

\footnotetext{
$\overline{{ }^{1} \text { Parseval's relation is more often seen in the literature as }\langle f, g\rangle_{L_{2}}}=\langle\hat{f}, \hat{g}\rangle_{L_{2}}$ with $\langle f, g\rangle_{L_{2}}:=\int f \bar{g}$.
} 
The weighted norm of a sequence $c \in \ell_{2, n}\left(\mathbb{Z}^{d}\right)$ is given by

$$
\|c\|_{\ell_{2, n}\left(\mathbb{Z}^{d}\right)}:=\left(\sum_{\boldsymbol{k} \in \mathbb{Z}^{d}}|c[\boldsymbol{k}]|^{2}\left(1+\|\boldsymbol{k}\|^{2}\right)^{n}\right)^{\frac{1}{2}} .
$$

\subsection{Fejér Kernel}

Let $\mathbb{T}$ denote the interval $[0,1]$. A function $f$ is 1-periodic if $f=f(\cdot+\boldsymbol{k})$, for all $\boldsymbol{k} \in$ $\mathbb{Z}^{d}$. Let $L_{1}\left(\mathbb{T}^{d}\right)$ be the space of all 1-periodic functions $f$ such that $\int_{\mathbb{T}^{d}}|f(\boldsymbol{x})| \mathrm{d} \boldsymbol{x}<\infty$. The Fourier coefficients of a function $f \in L_{1}\left(\mathbb{T}^{d}\right)$ are defined by

$$
\hat{f}[\boldsymbol{k}]:=\int_{\mathbb{T}^{d}} f(\boldsymbol{x}) \mathrm{e}^{-2 \pi \mathrm{j}\langle\boldsymbol{k}, \boldsymbol{x}\rangle} \mathrm{d} \boldsymbol{x}, \quad \boldsymbol{k} \in \mathbb{Z}^{d} .
$$

When studying the convergence of the Fourier series $\sum_{\boldsymbol{k} \in \mathbb{Z}^{d}} \hat{f}[\boldsymbol{k}] \mathrm{e}^{2 \pi \mathrm{j}\langle\boldsymbol{k}, \boldsymbol{x}\rangle}$ to $f(\boldsymbol{x})$, it is often useful to consider the (separable) $d$-dimensional Fejér kernel

$$
F_{N}(\boldsymbol{x}):=F_{N}^{(1)}\left(x_{1}\right) \cdots F_{N}^{(1)}\left(x_{d}\right), \quad N \geq 1,
$$

where the 1-dimensional Fejér kernel $F_{N}^{(1)}$ is defined as the Cesàro partial sum of the complex exponentials, i.e.,

$$
F_{N}^{(1)}(x):=\sum_{|k|<N}\left(1-\frac{|k|}{N}\right) \mathrm{e}^{2 \pi \mathrm{j} k x} .
$$

$F_{N}^{(1)}$ can also be expressed as

$$
F_{N}^{(1)}(x):=\frac{1}{N} \sum_{n=0}^{N-1} D_{n}^{(1)}(x),
$$

where $D_{n}^{(1)}(x):=\sum_{k=-n}^{n} \mathrm{e}^{2 \pi \mathrm{j} k x}$ is the 1-dimensional Dirichlet kernel. Putting (2) and (3) together, we write

$$
F_{N}(\boldsymbol{x})=\sum_{\|\boldsymbol{k}\|_{\infty}<N} \hat{F}_{N}[\boldsymbol{k}] \mathrm{e}^{2 \pi \mathrm{j}\langle\boldsymbol{k}, \boldsymbol{x}\rangle}
$$

where $\|\boldsymbol{k}\|_{\infty}:=\max _{1 \leq i \leq d}\left|k_{i}\right|$ and $\hat{F}_{N}[\boldsymbol{k}]$ is the $\boldsymbol{k}$-th Fourier coefficient of $F_{N}$ given by

$$
\hat{F}_{N}[\boldsymbol{k}]=\prod_{i=1}^{d}\left(1-\frac{\left|k_{i}\right|}{N}\right), \quad\|\boldsymbol{k}\|_{\infty}<N
$$


It is well-known $[15,36]$ that the Fejér kernel is a positive summability kernel with properties such as

- Periodicity $F_{N}(\boldsymbol{x}+\boldsymbol{k})=F_{N}(\boldsymbol{x}), \forall \boldsymbol{k} \in \mathbb{Z}^{d}$.

- Positivity $F_{N}(\boldsymbol{x}) \geq 0, \forall \boldsymbol{x} \in \mathbb{R}^{d}$.

- Normalization $\int_{\mathbb{T}^{d}} F_{N}(\boldsymbol{x}) \mathrm{d} \boldsymbol{x}=1, \forall N \geq 1$.

- Asymptotic sampling $\lim _{N \rightarrow \infty} \int_{\mathbb{T}^{d}} g(\boldsymbol{y}-\boldsymbol{x}) F_{N}(\boldsymbol{x}) \mathrm{d} \boldsymbol{x}=g(\boldsymbol{y}), \forall \boldsymbol{y} \in \mathbb{R}^{d}$, for all continuous functions $g$.

These properties are needed in the proof of Theorem 1.

\subsection{Distribution Theory}

In this section, we briefly review some important concepts of distribution theory; the standard reference for the subject is Schwartz' treatise [24], but more accessible texts are also available $[13,29,34]$. The theory of distributions generalizes the classical notion of functions to objects called distributions, characterized through their actions on well-behaved test functions. To be precise, let $\mathcal{D}\left(\mathbb{R}^{d}\right)$ denote the space of test functions that are infinitely differentiable and compactly supported. Test functions are also known as bump functions, and the space $\mathcal{D}\left(\mathbb{R}^{d}\right)$ is sometimes denoted by $\mathcal{C}_{\mathrm{c}}^{\infty}\left(\mathbb{R}^{d}\right)$. The space of distributions is defined as the dual space $\mathcal{D}^{\prime}\left(\mathbb{R}^{d}\right)$ of $\mathcal{D}\left(\mathbb{R}^{d}\right)$ that consists of all continuous linear functionals on $\mathcal{D}\left(\mathbb{R}^{d}\right)$. A distribution $f \in \mathcal{D}^{\prime}\left(\mathbb{R}^{d}\right)$ is specified by the mapping

$$
\begin{aligned}
f: \mathcal{D}\left(\mathbb{R}^{d}\right) & \rightarrow \mathbb{C} \\
\varphi & \mapsto\langle f, \varphi\rangle,
\end{aligned}
$$

where the scalar product notation $\langle f, \varphi\rangle$ (without the subscript $\mathbb{R}^{d}$ ) denotes the action of the functional $f$ on the test function $\varphi$. A regular type of distribution can be associated to a locally integrable function $f$, which is integrable on every compact subset of its domain, by defining

$$
\langle f, \varphi\rangle:=\int_{\mathbb{R}^{d}} f(\boldsymbol{x}) \varphi(\boldsymbol{x}) \mathrm{d} \boldsymbol{x} .
$$

By contrast, there are also irregular distributions that cannot be described by a scalar product with some function. The most famous example of irregular distributions is the Dirac pulse $\delta: \varphi \mapsto\langle\delta, \varphi\rangle=\varphi(\mathbf{0})$, which is often (mis)treated as an ordinary function in engineering texts.

For the sake of extending the Fourier transform, we are only interested in the space $\mathcal{S}^{\prime}\left(\mathbb{R}^{d}\right)$ of tempered distributions. This is the the dual space of Schwartz' space $\mathcal{S}\left(\mathbb{R}^{d}\right)$ of infinitely differentiable and rapidly decreasing functions. Specifically, a function $\varphi$ is included in $\mathcal{S}\left(\mathbb{R}^{d}\right)$ if it is infinitely differentiable and if

$$
\|\varphi\|_{k, \ell}=\sup _{x \in \mathbb{R}^{d}}\left|x^{k} \partial^{\ell} \varphi(x)\right|<\infty
$$


for every pair of multi-indices $\boldsymbol{k}, \boldsymbol{\ell} \in \mathbb{N}^{d}$. Such function $\varphi$ is called a Schwartzfunction and the quantities $\|\varphi\|_{k, \ell}$ are called the $S$ chwartz seminorms of $\varphi$. The space $\mathcal{S}\left(\mathbb{R}^{d}\right)$ equipped with this family of seminorms becomes a Fréchet space (complete metrizable locally convex space). In this space, a sequence of functions $\varphi_{n}$ converges to a function $\varphi$ if

$$
\left\|\varphi_{n}-\varphi\right\|_{k, \ell} \rightarrow 0 \quad \text { as } \quad n \rightarrow \infty, \forall \boldsymbol{k}, \ell \in \mathbb{N}^{d}
$$

The continuity of a tempered distribution $f \in \mathcal{S}^{\prime}\left(\mathbb{R}^{d}\right)$ means $\left\langle f, \varphi_{n}\right\rangle \rightarrow\langle f, \varphi\rangle$ whenever $\varphi_{n} \rightarrow \varphi$ in $\mathcal{S}\left(\mathbb{R}^{d}\right)$.

Since every test function is a Schwartz function, a tempered distribution is indeed a distribution. The advantage of using the space $\mathcal{S}\left(\mathbb{R}^{d}\right)$ is that the Fourier transform is an isomorphism on $\mathcal{S}\left(\mathbb{R}^{d}\right)$, which is not true on $\mathcal{D}\left(\mathbb{R}^{d}\right)$. With that, we can easily define the Fourier transform of a tempered distribution $f \in \mathcal{S}^{\prime}\left(\mathbb{R}^{d}\right)$ as another tempered distribution $\hat{f}=\mathcal{F} f$ given by

$$
\langle\hat{f}, \varphi\rangle=\langle\mathcal{F} f, \varphi\rangle:=\langle f, \mathcal{F} \varphi\rangle, \quad \forall \varphi \in \mathcal{S}\left(\mathbb{R}^{d}\right) .
$$

The above definition can be thought of as Parseval's relation for tempered distributions. As the Fourier transform is isomorphic on $\mathcal{S}\left(\mathbb{R}^{d}\right)$, it is also isomorphic on $\mathcal{S}^{\prime}\left(\mathbb{R}^{d}\right)$. The inverse Fourier transform operator on $\mathcal{S}^{\prime}\left(\mathbb{R}^{d}\right)$ is then given by

$$
\left\langle\mathcal{F}^{-1} f, \varphi\right\rangle=\left\langle f, \mathcal{F}^{-1} \varphi\right\rangle, \quad \forall \varphi \in \mathcal{S}\left(\mathbb{R}^{d}\right)
$$

For a multi-index $\ell \in \mathbb{N}^{d}$, the (weak) partial derivative $\partial^{\ell} f$ of a tempered distribution $f \in \mathcal{S}^{\prime}\left(\mathbb{R}^{d}\right)$ is also a tempered distribution defined by

$$
\left\langle\partial^{\ell} f, \varphi\right\rangle:=(-1)^{|\ell|}\left\langle f, \partial^{\ell} \varphi\right\rangle, \quad \forall \varphi \in \mathcal{S}\left(\mathbb{R}^{d}\right)
$$

The derivative defined above is called weak because it is also defined for (generalized) functions that are not differentiable in the ordinary sense, otherwise, the two notions are equivalent. It is important to mention here the differentiation property of the Fourier transform: for $f \in \mathcal{S}^{\prime}\left(\mathbb{R}^{d}\right)$ and $\boldsymbol{\ell} \in \mathbb{N}^{d}$, one has

$$
\mathcal{F}\left\{\partial^{\ell} f\right\}=(2 \pi \mathrm{j})^{|\ell|}(\cdot)^{\ell} \hat{f}
$$

In order to define the two other important operations on $\mathcal{S}^{\prime}\left(\mathbb{R}^{d}\right)$, namely, multiplication and convolution, we need to consider the two spaces $\mathcal{O}_{M}\left(\mathbb{R}^{d}\right)$ and $\mathcal{O}_{C}^{\prime}\left(\mathbb{R}^{d}\right)$. In particular, a function $\varphi$ is in $\mathcal{O}_{M}\left(\mathbb{R}^{d}\right)$ if it is infinitely differentiable and not growing faster than some polynomial, whereas a tempered distribution $f$ is in $\mathcal{O}_{C}^{\prime}\left(\mathbb{R}^{d}\right)$ if $\hat{f} \in \mathcal{O}_{M}\left(\mathbb{R}^{d}\right)$. Note that, for all $\psi \in \mathcal{O}_{M}\left(\mathbb{R}^{d}\right)$ and $\varphi \in \mathcal{S}\left(\mathbb{R}^{d}\right)$, we have that $\psi \varphi \in \mathcal{S}\left(\mathbb{R}^{d}\right)$. Thus, we can define the multiplication and convolution with a tempered distribution $f \in \mathcal{S}^{\prime}\left(\mathbb{R}^{d}\right)$ by 


$$
\begin{aligned}
\langle f \cdot \psi, \varphi\rangle & :=\langle f, \psi \varphi\rangle, \quad \forall \psi \in \mathcal{O}_{M}\left(\mathbb{R}^{d}\right), \varphi \in \mathcal{S}\left(\mathbb{R}^{d}\right) \\
\text { and }\langle f * g, \varphi\rangle & :=\langle f, g(-\cdot) * \varphi\rangle, \quad \forall g \in \mathcal{O}_{C}^{\prime}\left(\mathbb{R}^{d}\right), \varphi \in \mathcal{S}\left(\mathbb{R}^{d}\right),
\end{aligned}
$$

respectively. Importantly, these definitions retain the classical duality between multiplication and convolution so that, for $f \in \mathcal{S}^{\prime}\left(\mathbb{R}^{d}\right)$,

$$
\begin{aligned}
& \mathcal{F}(f * g)=\mathcal{F} f \cdot \mathcal{F} g, \quad \forall g \in \mathcal{O}_{C}^{\prime}\left(\mathbb{R}^{d}\right) ; \\
& \mathcal{F}(f \cdot \psi)=\mathcal{F} f * \mathcal{F} \psi, \quad \forall \psi \in \mathcal{O}_{M}\left(\mathbb{R}^{d}\right) .
\end{aligned}
$$

In the remainder of the paper, we also need the translation operator on $\mathcal{S}^{\prime}\left(\mathbb{R}^{d}\right)$, which corresponds to

$$
\left\langle f\left(\cdot+\boldsymbol{x}_{0}\right), \varphi\right\rangle=\left\langle f, \varphi\left(\cdot-\boldsymbol{x}_{0}\right)\right\rangle, \quad \text { for } f \in \mathcal{S}^{\prime}\left(\mathbb{R}^{d}\right), \varphi \in \mathcal{S}\left(\mathbb{R}^{d}\right), \boldsymbol{x}_{0} \in \mathbb{R}^{d} \text {. }
$$

\section{PSF for Polynomially Growing Functions}

We are now ready to state the first generalization of the PSF for continuous functions of polynomial growth. Note that the periodization of the Fourier transform of the function is interpreted, in this case, as a Cesàro sum.

Theorem 1 (PSF for polynomially growing functions) If $f$ is a continuous function of polynomial growth, then

$$
\sum_{\boldsymbol{k} \in \mathbb{Z}^{d}} f(\boldsymbol{k}) \mathrm{e}^{-2 \pi \mathrm{j}\langle\boldsymbol{k}, \cdot\rangle}=\lim _{N \rightarrow \infty} \sum_{\|\ell\|_{\infty}<N} \hat{F}_{N}[\ell] \hat{f}(\cdot+\boldsymbol{\ell}) \quad \text { in } \mathcal{S}^{\prime}\left(\mathbb{R}^{d}\right)
$$

where $\hat{F}_{N}[\ell]=\prod_{i=1}^{d}\left(1-\left|\ell_{i}\right| / N\right)$ is the $\ell$-th Fourier coefficient of the Fejér kernel.

Proof We shall show that both sides of (7) are tempered distributions that are equal when acting on any test function $\varphi \in \mathcal{S}\left(\mathbb{R}^{d}\right)$. On one hand, the action of the LHS on $\varphi$ is given by

$$
\begin{aligned}
\left\langle\sum_{\boldsymbol{k} \in \mathbb{Z}^{d}} f(\boldsymbol{k}) \mathrm{e}^{-2 \pi \mathrm{j}\langle\boldsymbol{k}, \cdot\rangle}, \varphi\right\rangle & =\sum_{\boldsymbol{k} \in \mathbb{Z}^{d}} f(\boldsymbol{k})\left\langle\mathrm{e}^{-2 \pi \mathrm{j}\langle\boldsymbol{k}, \cdot\rangle}, \varphi\right\rangle_{\mathbb{R}^{d}}=\sum_{\boldsymbol{k} \in \mathbb{Z}^{d}} f(\boldsymbol{k}) \hat{\varphi}(\boldsymbol{k}) \\
& =\sum_{\boldsymbol{k} \in \mathbb{Z}^{d}} g(\boldsymbol{k}),
\end{aligned}
$$

where $g:=f \hat{\varphi}$. The sum in (8) converges absolutely since $\hat{\varphi} \in \mathcal{S}\left(\mathbb{R}^{d}\right)$ and since

$$
\sum_{\boldsymbol{k} \in \mathbb{Z}^{d}}|f(\boldsymbol{k}) \hat{\varphi}(\boldsymbol{k})| \leq C \sum_{\boldsymbol{k} \in \mathbb{Z}^{d}}(1+\|\boldsymbol{k}\|)^{n}|\hat{\varphi}(\boldsymbol{k})|<\infty
$$

where we have used the fact that $|f(\boldsymbol{x})| \leq C(1+\|\boldsymbol{x}\|)^{n}$, for all $\boldsymbol{x} \in \mathbb{R}^{d}$ and for some $n \in \mathbb{N}$. 
On the other hand, since $f$ is bounded by a polynomial and $\hat{\varphi}$ is rapidly decaying, the action of the RHS of (7) on $\varphi$ can be written as

$$
\begin{aligned}
\left\langle\lim _{N \rightarrow \infty} \sum_{\|\ell\|_{\infty}<N} \hat{F}_{N}[\ell] \hat{f}(\cdot+\ell), \varphi\right\rangle & =\lim _{N \rightarrow \infty} \sum_{\|\ell\|_{\infty}<N} \hat{F}_{N}[\ell]\langle\hat{f}(\cdot+\boldsymbol{\ell}), \varphi\rangle \\
& =\lim _{N \rightarrow \infty} \sum_{\|\ell\|_{\infty}<N} \hat{F}_{N}[\ell]\langle f, \mathcal{F}\{\varphi(\cdot-\boldsymbol{\ell})\}\rangle \\
& =\lim _{N \rightarrow \infty} \sum_{\|\ell\|_{\infty}<N} \hat{F}_{N}[\boldsymbol{\ell}] \int_{\mathbb{R}^{d}} f(\boldsymbol{x}) \hat{\varphi}(\boldsymbol{x}) \mathrm{e}^{-2 \pi \mathrm{j}\langle\ell, \boldsymbol{x}\rangle} \mathrm{d} \boldsymbol{x} \\
& =\lim _{N \rightarrow \infty} \int_{\mathbb{R}^{d}} g(\boldsymbol{x}) \sum_{\|\boldsymbol{\ell}\|_{\infty}<N} \hat{F}_{N}[\boldsymbol{\ell}] \mathrm{e}^{-2 \pi \mathrm{j}\langle\boldsymbol{\ell}, \boldsymbol{x}\rangle} \mathrm{d} \boldsymbol{x} \\
& =\lim _{N \rightarrow \infty} \int_{\mathbb{R}^{d}} g(-\boldsymbol{x}) F_{N}(\boldsymbol{x}) \mathrm{d} \boldsymbol{x} \\
& =\lim _{N \rightarrow \infty} \sum_{\boldsymbol{k} \in \mathbb{Z}^{d}} \int_{\mathbb{T}^{d}} g(\boldsymbol{k}-\boldsymbol{x}) F_{N}(\boldsymbol{x}) \mathrm{d} \boldsymbol{x} .
\end{aligned}
$$

Note that, for each $k \in \mathbb{Z}^{d}$, the integral in (10) is bounded by

$$
\left|\int_{\mathbb{T}^{d}} g(\boldsymbol{k}-\boldsymbol{x}) F_{N}(\boldsymbol{x}) \mathrm{d} \boldsymbol{x}\right| \leq \sup _{\boldsymbol{x} \in \mathbb{T}^{d}}|g(\boldsymbol{k}-\boldsymbol{x})| \cdot \int_{\mathbb{T}^{d}}\left|F_{N}(\boldsymbol{x})\right| \mathrm{d} \boldsymbol{x}=\sup _{\boldsymbol{x} \in \mathbb{T}^{d}}|g(\boldsymbol{k}-\boldsymbol{x})|,
$$

where we have used the continuity of $g$ and the fact that $F_{N}(\boldsymbol{x})$ is a positive kernel that integrates to unity on $\mathbb{T}^{d}$. As $g=f \hat{\varphi}$ decays faster than any polynomial, it must be that the sequence $\left\{\sup _{\boldsymbol{x} \in \mathbb{T}^{d}}|g(\boldsymbol{k}-\boldsymbol{x})|\right\}_{\boldsymbol{k} \in \mathbb{Z}^{d}}$ is absolutely summable. Therefore, we can invoke Lebesgue's dominated-convergence theorem to exchange the limit and sum in (10) as

$$
\begin{aligned}
\left\langle\lim _{N \rightarrow \infty} \sum_{\|\ell\|_{\infty}<N} \hat{F}_{N}[\ell] \hat{f}(\cdot+\boldsymbol{\ell}), \varphi\right\rangle & =\sum_{\boldsymbol{k} \in \mathbb{Z}^{d}} \lim _{N \rightarrow \infty} \int_{\mathbb{T}^{d}} g(\boldsymbol{k}-\boldsymbol{x}) F_{N}(\boldsymbol{x}) \mathrm{d} \boldsymbol{x} \\
& =\sum_{\boldsymbol{k} \in \mathbb{Z}^{d}} g(\boldsymbol{k})
\end{aligned}
$$

where we have used the asymptotic-sampling property of the Fejér kernel $F_{N}(\boldsymbol{x})$. Equating (8) and (12) gives us the desired identity (7) in the space of tempered distributions $\mathcal{S}^{\prime}\left(\mathbb{R}^{d}\right)$. 


\section{PSF on Weighted Sobolev Spaces}

\subsection{Weighted Sobolev Spaces}

When sampling in weighted- $L_{2}$ spaces, we need to impose some order of differentiability on the signals by considering weighted Sobolev spaces. To that end, we first define the (unweighted) Sobolev spaces of integer orders $k \in \mathbb{N}$ as

$$
H_{2}^{k}\left(\mathbb{R}^{d}\right):=\left\{f \in \mathcal{S}^{\prime}\left(\mathbb{R}^{d}\right): \partial^{\ell} f \in L_{2}\left(\mathbb{R}^{d}\right), \forall|\ell| \leq k\right\}
$$

where $\partial^{\ell} f$ is the $\ell$-th weak derivative of $f$ defined in (5). In words, the Sobolev space of order $k$ consists of functions whose weak derivatives up to order $k$ are all in $L_{2}\left(\mathbb{R}^{d}\right)$. The Sobolev norm of a function $f \in H_{2}^{k}\left(\mathbb{R}^{d}\right)$ is given by

$$
\|f\|_{H_{2}^{k}\left(\mathbb{R}^{d}\right)}:=\sum_{|\ell| \leq k}\left\|\partial^{\ell} f\right\|_{L_{2}\left(\mathbb{R}^{d}\right)} .
$$

Meanwhile, the Sobolev spaces of fractional orders $s \in \mathbb{R}$ are defined via the Fourier transform as

$$
L_{2}^{s}\left(\mathbb{R}^{d}\right):=\left\{f \in \mathcal{S}^{\prime}\left(\mathbb{R}^{d}\right): J^{s} f \in L_{2}\left(\mathbb{R}^{d}\right)\right\}
$$

where the operator $J^{s}$ is defined as

$$
J^{s} f:=\mathcal{F}^{-1}\left\{\left(1+\|\cdot\|^{2}\right)^{\frac{s}{2}} \hat{f}\right\}
$$

The Sobolev norm of an element $f \in L_{2}^{s}\left(\mathbb{R}^{d}\right)$ is given by

$$
\|f\|_{L_{2}^{s}\left(\mathbb{R}^{d}\right)}:=\left\|J^{s} f\right\|_{L_{2}\left(\mathbb{R}^{d}\right)} .
$$

We want to emphasize that definition (15) is indeed an extension of definition (13): one can show by using Fourier multipliers $[9,10]$ that, for $k \in \mathbb{N}$, the spaces $H_{2}^{k}\left(\mathbb{R}^{d}\right)$ and $L_{2}^{k}\left(\mathbb{R}^{d}\right)$ are identical and their corresponding norms given in (14) and (17) are equivalent.

We now extend (15) to define the weighted Sobolev spaces (of fractional orders) as

$$
L_{2, n}^{s}\left(\mathbb{R}^{d}\right):=\left\{f \in \mathcal{S}^{\prime}\left(\mathbb{R}^{d}\right): J^{s} f \in L_{2, n}\left(\mathbb{R}^{d}\right)\right\}, \quad s \in \mathbb{R}, n \in \mathbb{Z} .
$$

The weighted Sobolev norm of an element $f \in L_{2, n}^{s}\left(\mathbb{R}^{d}\right)$ is given by

$$
\|f\|_{L_{2, n}^{s}\left(\mathbb{R}^{d}\right)}:=\left\|J^{s} f\right\|_{L_{2, n}\left(\mathbb{R}^{d}\right)} .
$$


It is also useful to introduce the Bessel potential kernels

$$
\beta^{(s)}:=\mathcal{F}^{-1}\left\{\left(1+\|\cdot\|^{2}\right)^{-s / 2}\right\}, \quad s \in \mathbb{R} .
$$

Informally speaking, the operator $J^{s}$ accumulates in some sense all the derivatives up to order $s$. Therefore, $s$ is referred to as the order of differentiability and an element of the space $L_{2, n}^{s}\left(\mathbb{R}^{d}\right)$ is said to have $s$ derivatives that are bounded by a polynomial of degree $n$ in the $L_{2}$ sense. Since any $f \in L_{2, n}^{s}\left(\mathbb{R}^{d}\right)$ can be written as

$$
f=\left(J^{s} f\right) * \beta^{(s)},
$$

we can say, in the language of signal processing, that a signal lives in the weighted Sobolev space of order $s$ if it is the result of filtering a weighted- $L_{2}$ signal with the Bessel potential kernel of order $s$.

For a deeper understanding of Sobolev spaces, interested readers are referred to [2, 9,17]; excellent resources are also available online, see, for example, [28]. The Bessel potentials were discussed thoroughly in $[1,3,26]$. Results regarding the sampling of growing signals in weighted Sobolev spaces can be found in [18]. Proposition 1 asserts that elements of the weighted Sobolev space $L_{2, n}^{s}\left(\mathbb{R}^{d}\right)$ are polynomially growing functions when the order of differentiability $s$ exceeds the threshold $d / 2$.

Proposition 1 If $f$ is a function in the weighted Sobolev space $L_{2,-n}^{s}\left(\mathbb{R}^{d}\right)$ with $s>$ $d / 2$ and $n \in \mathbb{N}$, then $f$ is polynomially growing.

Proof We first write $f=\left(J^{s} f\right) * \beta^{(s)}$. Since $f \in L_{2,-n}^{s}\left(\mathbb{R}^{d}\right)$, it must be that $J^{s} f \in$ $L_{2,-n}\left(\mathbb{R}^{d}\right)$. Furthermore, from [18, Proposition7], we also know that $\beta^{(s)} \in L_{2, n}\left(\mathbb{R}^{d}\right)$. It then follows from [18, Proposition 1] that the convolution $\left(J^{s} f\right) * \beta^{(s)}$ is a continuous function that is bounded pointwise by the polynomial $C(1+\|\boldsymbol{x}\|)^{n}$. Thus, $f$ is a polynomially growing function.

\subsection{PSF on Weighted Sobolev Spaces}

By Proposition 1, the weighted Sobolev space $L_{2,-n}^{s}\left(\mathbb{R}^{d}\right)$, with $s>d / 2, n \in \mathbb{N}$, is embedded in the space of polynomially growing functions. Therefore, as shown in Theorem 1 , PSF automatically holds in $\mathcal{S}^{\prime}\left(\mathbb{R}^{d}\right)$ for every function $f \in L_{2,-n}^{s}\left(\mathbb{R}^{d}\right)$, where the periodization of $\hat{f}$ converges conditionally in the sense of the Cesàro summation. In this section, we show that PSF actually holds in a stronger sense for functions in a particular weighted Sobolev space. Specifically, PSF is true in the negative-order Sobolev space $L_{2}^{-2 n}\left(\mathbb{R}^{d}\right)$ for every $f \in L_{2,-2 n}^{s}$, where $s>d / 2$ and $n \in \mathbb{N}$. With this stricter requirement on the function $f$, the periodization of $\hat{f}$ converges unconditionally, and so there is no need to use the Cesàro summation. The equality in PSF now means that the Fourier transforms of both sides are growing functions that are equal almost everywhere. The central result of this section is stated in Theorem 4; but, before that, we need several auxiliary results: Theorem 2, which was presented in [18], 
makes sure that the sampling is bounded in weighted Sobolev spaces with sufficient order of differentiability. Theorem 3 shows that the periodization in the RHS of PSF is a well-defined distribution.

Theorem 2 If $f$ is a continuous function that is included in the weighted Sobolev space $L_{2, n}^{s}\left(\mathbb{R}^{d}\right)$ with $s>d / 2$ and $n \in \mathbb{Z}$, then the sampled sequence $\{f(\boldsymbol{k})\}_{\boldsymbol{k} \in \mathbb{Z}^{d}}$ is an element of $\ell_{2, n}\left(\mathbb{Z}^{d}\right)$ and we have that

$$
\|f[\cdot]\|_{\ell_{2, n}\left(\mathbb{Z}^{d}\right)} \leq C_{n, s}\|f\|_{L_{2, n}^{s}\left(\mathbb{R}^{d}\right)} .
$$

Proof See [18, Theorem3] for a proof for $n \leq 0$. When $n>0$, the same result is obtained by using duality.

Theorem 3 Let $f \in L_{2,-2 n}^{s}\left(\mathbb{R}^{d}\right)$ with $s>d / 2$ and $n \in \mathbb{N}$. Then, the series $\sum_{\boldsymbol{k} \in \mathbb{Z}^{d}}\langle\hat{f}(\cdot+\boldsymbol{k}), \varphi\rangle$ is absolutely convergent for every test function $\varphi \in \mathcal{C}_{\mathrm{c}}^{\infty}\left(\mathbb{R}^{d}\right)$ and we have that

$$
\sum_{\boldsymbol{k} \in \mathbb{Z}^{d}}|\langle\hat{f}(\cdot+\boldsymbol{k}), \varphi\rangle| \leq C_{n, s}\|f\|_{L_{2,-2 n}^{s}\left(\mathbb{R}^{d}\right)}\|\varphi\|_{L_{2}^{2 n}\left(\mathbb{R}^{d}\right)} .
$$

Proof See Appendix.

Theorem 4 (PSF on weighted Sobolev spaces) If $f$ is a continuous function in the weighted Sobolev space $L_{2,-2 n}^{s}\left(\mathbb{R}^{d}\right)$ with $s>d / 2$ and $n \in \mathbb{N}$, then

$$
\sum_{\boldsymbol{k} \in \mathbb{Z}^{d}} f(\boldsymbol{k}) \mathrm{e}^{-2 \pi \mathrm{j}\langle\boldsymbol{k}, \cdot\rangle}=\sum_{\boldsymbol{\ell} \in \mathbb{Z}^{d}} \hat{f}(\cdot+\boldsymbol{\ell}) \quad \text { in } L_{2}^{-2 n}\left(\mathbb{R}^{d}\right)
$$

Proof We first recall the well-known result [5, Corollary 6.2.8] that $L_{2}^{-2 n}\left(\mathbb{R}^{d}\right)=$ $\left(L_{2}^{2 n}\left(\mathbb{R}^{d}\right)\right)^{\prime}$. Therefore, it suffices to show that the two sides of (22) are identical in the dual space of $L_{2}^{2 n}\left(\mathbb{R}^{d}\right)$.

Let us fix a test function $\varphi \in \mathcal{C}_{\mathrm{c}}^{\infty}\left(\mathbb{R}^{d}\right)$. As $f$ is polynomially growing (according to Proposition 1) and $\varphi \in \mathcal{S}\left(\mathbb{R}^{d}\right)$, then, similarly to the proof of Theorem 1 , the action of the LHS of (22) on $\varphi$ is well defined by

$$
\left\langle\sum_{\boldsymbol{k} \in \mathbb{Z}^{d}} f(\boldsymbol{k}) \mathrm{e}^{-2 \pi \mathrm{j}\langle\boldsymbol{k}, \cdot\rangle}, \varphi\right\rangle=\sum_{\boldsymbol{k} \in \mathbb{Z}^{d}} f(\boldsymbol{k})\left\langle\mathrm{e}^{-2 \pi \mathrm{j}\langle\boldsymbol{k}, \cdot\rangle}, \varphi\right\rangle_{\mathbb{R}^{d}}=\sum_{\boldsymbol{k} \in \mathbb{Z}^{d}} g(\boldsymbol{k}),
$$


where $g:=f \hat{\varphi}$ is a continuous rapidly decaying function. Furthermore, from Theorem 2 and by the Cauchy-Schwarz inequality, we have the bound

$$
\begin{aligned}
\left|\left\langle\sum_{\boldsymbol{k} \in \mathbb{Z}^{d}} f(\boldsymbol{k}) \mathrm{e}^{-2 \pi j\langle\boldsymbol{k}, \cdot\rangle}, \varphi\right\rangle\right| & \leq \sum_{\boldsymbol{k} \in \mathbb{Z}^{d}}|f(\boldsymbol{k}) \hat{\varphi}(\boldsymbol{k})| \\
& \leq\|f[\cdot]\|_{\ell_{2,-2 n}\left(\mathbb{Z}^{d}\right)}\|\hat{\varphi}[\cdot]\|_{\ell_{2,2 n}\left(\mathbb{Z}^{d}\right)} \\
& \leq C_{n, s}\|f\|_{L_{2,-2 n}^{s}\left(\mathbb{R}^{d}\right)}\|\hat{\varphi}\|_{L_{2,2 n}^{s}\left(\mathbb{R}^{d}\right)} \\
& \leq C_{n, s}\|f\|_{L_{2,-2 n}^{s}\left(\mathbb{R}^{d}\right)}\|\varphi\|_{L_{2}^{2 n}\left(\mathbb{R}^{d}\right)} .
\end{aligned}
$$

On the other hand, the action of the RHS of (22) on $\varphi$ is now interpreted as

$$
\left\langle\sum_{\ell \in \mathbb{Z}^{d}} \hat{f}(\cdot+\boldsymbol{\ell}), \varphi\right\rangle=\sum_{\ell \in \mathbb{Z}^{d}}\langle\hat{f}(\cdot+\ell), \varphi\rangle,
$$

which is finite from Theorem 3 .

Let us consider the periodization $h(\boldsymbol{x}):=\sum_{\boldsymbol{k} \in \mathbb{Z}^{d}} g(\boldsymbol{x}+\boldsymbol{k})$, whose Fourier coefficients are given by

$$
\begin{aligned}
\hat{h}[\ell] & =\int_{\mathbb{T}^{d}} \sum_{\boldsymbol{k} \in \mathbb{Z}^{d}} g(\boldsymbol{x}+\boldsymbol{k}) \mathrm{e}^{-2 \pi \mathrm{j}\langle\boldsymbol{\ell}, \boldsymbol{x}\rangle} \mathrm{d} \boldsymbol{x} \\
& =\sum_{\boldsymbol{k} \in \mathbb{Z}^{d}} \int_{\mathbb{T}^{d}} g(\boldsymbol{x}+\boldsymbol{k}) \mathrm{e}^{-2 \pi \mathrm{j}\langle\ell, \boldsymbol{x}+\boldsymbol{k}\rangle} \mathrm{d} \boldsymbol{x} \\
& =\int_{\mathbb{R}^{d}} g(\boldsymbol{x}) \mathrm{e}^{-2 \pi \mathrm{j}\langle\boldsymbol{\ell}, \boldsymbol{x}\rangle} \mathrm{d} \boldsymbol{x},
\end{aligned}
$$

where the exchange of integral and sum is justified by the dominated convergence theorem and by the fact that $g \in L_{1}\left(\mathbb{R}^{d}\right)$. With the same manipulation as in the proof of Theorem 1 , we obtain

$$
\hat{h}[\ell]=\langle\hat{f}(\cdot+\ell), \varphi\rangle
$$

which, together with Theorem 3, establishes that the Fourier coefficients of $h$ are absolutely summable. We can also show that $h(\boldsymbol{x})$ is continuous at $\mathbf{0}$. Indeed, as $g$ is rapidly decaying, for any $\varepsilon>0$, there exists $N \in \mathbb{N}$ such that

$$
\sum_{\|\boldsymbol{k}\|>N}|g(\boldsymbol{x}+\boldsymbol{k})|<\frac{\varepsilon}{3}, \quad \forall\|\boldsymbol{x}\|<1 .
$$

Moreover, since $g$ is continuous, the finite sum $\sum_{\|\boldsymbol{k}\| \leq N} g(\cdot+\boldsymbol{k})$ is continuous. Thus, there exists $\delta>0$ such that 


$$
\left|\sum_{\|\boldsymbol{k}\| \leq N}(g(\boldsymbol{x}+\boldsymbol{k})-g(\boldsymbol{k}))\right|<\frac{\varepsilon}{3}, \quad \forall\|\boldsymbol{x}\|<\delta .
$$

Hence, for every $\|\boldsymbol{x}\|<\min (1, \delta)$, we have that

$$
\begin{aligned}
\left|\sum_{\boldsymbol{k} \in \mathbb{Z}^{d}}(g(\boldsymbol{x}+\boldsymbol{k})-g(\boldsymbol{k}))\right| \leq & \left|\sum_{\|\boldsymbol{k}\| \leq N}(g(\boldsymbol{x}+\boldsymbol{k})-g(\boldsymbol{k}))\right|+\sum_{\|\boldsymbol{k}\|>N}|g(\boldsymbol{x}+\boldsymbol{k})| \\
& +\sum_{\|\boldsymbol{k}\|>N}|g(\boldsymbol{k})|<\varepsilon,
\end{aligned}
$$

which implies the continuity of $h$ at $\mathbf{0}$. Therefore, the Fourier series of $h$ at $\boldsymbol{x}=\mathbf{0}$ converges to

$$
h(\mathbf{0})=\sum_{\ell \in \mathbb{Z}^{d}} \hat{h}[\ell]
$$

Combining this with (23), (25), (26) yields

$$
\left\langle\sum_{\boldsymbol{k} \in \mathbb{Z}^{d}} f(\boldsymbol{k}) \mathrm{e}^{-2 \pi \mathrm{j}\langle\boldsymbol{k}, \cdot\rangle}, \varphi\right\rangle=\left\langle\sum_{\boldsymbol{\ell} \in \mathbb{Z}^{d}} \hat{f}(\cdot+\boldsymbol{\ell}), \varphi\right\rangle, \quad \forall \varphi \in \mathcal{C}_{\mathrm{c}}^{\infty}\left(\mathbb{R}^{d}\right) .
$$

The final step of the proof is to extend the validity of (27) to all $\varphi \in L_{2}^{2 n}\left(\mathbb{R}^{d}\right)$. From bounds (21) and (24) and by the Hahn-Banach theorem [23, Theorem3.3], each side of (22) can be extended to a continuous linear functional on $L_{2}^{2 n}\left(\mathbb{R}^{d}\right)$. (Note that, for linear operators between normed spaces, boundedness is equivalent to continuity.) We also know that $\mathcal{C}_{\mathrm{c}}^{\infty}\left(\mathbb{R}^{d}\right)$ is a dense subspace of $L_{2}^{2 n}\left(\mathbb{R}^{d}\right)$, as a consequence of the Meyers-Serrin theorem [17, Theorem 10.15]. Hence, the continuous extensions on both sides must be identical on the whole space $L_{2}^{2 n}\left(\mathbb{R}^{d}\right)$, thus completing the proof.

\section{Discussion}

We have derived two generalizations of the Poisson summation formula, one for continuous functions of polynomial growth and the other for functions in some weighted Sobolev space $L_{2,-2 n}^{s}\left(\mathbb{R}^{d}\right)$ with $s>d / 2$ and $n \in \mathbb{N}$. In the first generalization, with the minimal assumption on the continuity and the polynomial growth of the function, the PSF identity holds in the space of tempered distributions $\mathcal{S}^{\prime}\left(\mathbb{R}^{d}\right)$, where the periodization on the RHS of the formula converges only conditionally in the sense of the Cesàro summation. The second generalization gives a stronger sense of equality when the function is restricted to the space $L_{2,-2 n}^{s}\left(\mathbb{R}^{d}\right)$ which is embedded in the space of polynomially growing functions provided that $s>d / 2$. With this assumption, the PSF identity holds in the negative-order Sobolev space $L_{2}^{-2 n}\left(\mathbb{R}^{d}\right)$, which is a subspace 
of $\mathcal{S}^{\prime}\left(\mathbb{R}^{d}\right)$. Furthermore, both sides of the formula converge unconditionally without using any summability methods.

We conclude the paper by demonstrating the application of the two generalized PSFs to some simple growing signals. Let us apply the generalized PSFs to the onesided power functions $x_{+}^{\alpha}$ mentioned in the introduction section, for some particular values of $\alpha>0$. Recall [30] that the (distributional) Fourier transform of $x_{+}^{\alpha}$ is given by

$$
\mathcal{F}\left\{x_{+}^{\alpha}\right\}(\xi)= \begin{cases}\frac{\Gamma(\alpha+1)}{(2 \pi \mathrm{j} \xi)^{\alpha+1}}, & \text { if } \alpha>0, \alpha \notin \mathbb{N} \\ \frac{n !}{(2 \pi \mathrm{j} \xi)^{n+1}}+\frac{1}{2}\left(\frac{\mathrm{j}}{2 \pi}\right)^{n} \delta^{(n)}(\xi), & \text { if } \alpha=n \in \mathbb{N}\end{cases}
$$

where $\Gamma$ is Euler's gamma function and $\delta^{(n)}$ is the $n$th derivative of the Dirac pulse. Obviously, $x_{+}^{\alpha}$ is continuous and polynomially growing for all $\alpha>0$. In particular, when $\alpha=1 / 2$, the first generalized PSF yields

$$
\sum_{k \in \mathbb{N}} \sqrt{k} \mathrm{e}^{-2 \pi \mathrm{j} k \xi}=\lim _{N \rightarrow \infty} \sum_{|\ell|<N}\left(1-\frac{|\ell|}{N}\right) \frac{\sqrt{\pi} / 2}{(2 \pi \mathrm{j}(\xi+\ell))^{3 / 2}} \text { in } \mathcal{S}^{\prime}(\mathbb{R})
$$

When $\alpha=1$, both $x_{+}$and its weak derivative, which is the Heaviside step function, are bounded by the polynomial $1+x^{2}$ in the $L_{2}$ sense. This implies that $x_{+} \in L_{2,-2}^{1}(\mathbb{R})$. Thus, the second generalized PSF gives

$$
\sum_{k \in \mathbb{N}} k \mathrm{e}^{-2 \pi \mathrm{j} k \xi}=\frac{\mathrm{j}}{4 \pi} \sum_{\ell \in \mathbb{Z}} \delta^{\prime}(\xi+\ell)-\frac{1}{4 \pi^{2}} \sum_{\ell \in \mathbb{Z}} \frac{1}{(\xi+\ell)^{2}} \text { in } L_{2}^{-2}(\mathbb{R}) .
$$

Although the validity of formulas (28) and (29) are guaranteed by Theorems 1 and 4, respectively, their interpretations need further investigation.

Acknowledgements The authors thank Prof. Hans Georg Feichtinger for his interest in the topic and for helpful discussions, especially for bringing to our attention the references [34,35]. We also thank the anonymous reviewers for their constructive comments that greatly improved the paper. This research was funded by the Swiss National Science Foundation under Grant 200020-162343.

\section{Appendix: Proof of Theorem 3}

Before starting the proof of Theorem 3, we need a technical lemma.

Lemma 1 For any $r \in \mathbb{R}$ and $\boldsymbol{k} \in \mathbb{N}^{d}$, there exists a constant $C_{r, \boldsymbol{k}}$ depending on $r$ and $\boldsymbol{k}$ such that

$$
\left|\partial^{k}\left(1+\|\boldsymbol{\xi}\|^{2}\right)^{r}\right| \leq C_{r, \boldsymbol{k}}\left(1+\|\boldsymbol{\xi}\|^{2}\right)^{r}, \quad \forall \boldsymbol{\xi} \in \mathbb{R}^{d}
$$


Proof of Lemma 1 It suffices to show, by induction, that

$$
\partial^{k}\left(1+\|\boldsymbol{\xi}\|^{2}\right)^{r}=\sum_{n=0}^{|\boldsymbol{k}|} P_{n}(\boldsymbol{\xi})\left(1+\|\boldsymbol{\xi}\|^{2}\right)^{r-n}, \quad \forall \boldsymbol{k} \in \mathbb{N}^{d}
$$

where each term $P_{n}(\xi)$ satisfies $\left|\partial^{\ell} P_{n}(\xi)\right| \leq C_{r, \ell}\left(1+\|\xi\|^{2}\right)^{n}, \forall \ell \in \mathbb{N}^{d}$. Obviously, the claim is true for $\boldsymbol{k}=\mathbf{0}$ by choosing $P_{0}(\xi)=1$. Suppose (30) is true for some $\boldsymbol{k} \in \mathbb{N}^{d}$, we shall show that it is also true for $\boldsymbol{k}^{\prime}=\left(k_{1}, \ldots, k_{i}+1, \ldots, k_{d}\right)$ for arbitrary $i$. Indeed, from the induction hypothesis and by Leibniz' rule, we have that

$$
\begin{aligned}
\partial^{\boldsymbol{k}^{\prime}}\left(1+\|\boldsymbol{\xi}\|^{2}\right)^{r} & =\frac{\partial}{\partial \xi_{i}} \sum_{n=0}^{|\boldsymbol{k}|} P_{n}(\boldsymbol{\xi})\left(1+\|\boldsymbol{\xi}\|^{2}\right)^{r-n} \\
& =\sum_{n=0}^{|\boldsymbol{k}|}\left(\frac{\partial}{\partial \xi_{i}} P_{n}(\xi)\left(1+\|\xi\|^{2}\right)^{r-n}+2(r-n) \xi_{i} P_{n}(\xi)\left(1+\|\xi\|^{2}\right)^{r-n-1}\right) \\
& =\sum_{n=0}^{\left|\boldsymbol{k}^{\prime}\right|} Q_{n}(\xi)\left(1+\|\boldsymbol{\xi}\|^{2}\right)^{r-n}
\end{aligned}
$$

where

$$
Q_{n}(\xi):= \begin{cases}\frac{\partial}{\partial \xi_{i}} P_{n}(\xi), & n=0 \\ \frac{\partial}{\partial \xi_{i}} P_{n}(\xi)+2(r-n+1) \xi_{i} P_{n-1}(\xi), & n=1, \ldots,|\boldsymbol{k}| \\ 2(r-n+1) \xi_{i} P_{n-1}(\xi) & n=|\boldsymbol{k}|+1 .\end{cases}
$$

From the induction hypothesis and from the simple inequality $2\left|\xi_{i}\right| \leq 1+\|\xi\|^{2}$, it is easy to check that, for each $n \leq\left|\boldsymbol{k}^{\prime}\right|, Q_{n}(\boldsymbol{\xi})$ satisfies the property $\left|\partial^{\ell} Q_{n}(\boldsymbol{\xi})\right| \leq$ $C_{r, \ell}\left(1+\|\xi\|^{2}\right)^{n}, \forall \ell \in \mathbb{N}^{d}$. This completes the proof of Lemma 1 .

Proof of Theorem 3 Let $w:=\left(1+\|\cdot\|^{2}\right)^{n}$ be the weighting function. Let $\mathbb{B}$ denote the support of $\varphi$. For each $\boldsymbol{k} \in \mathbb{Z}^{d}$, we define $\varphi_{\boldsymbol{k}}:=\varphi(\cdot-\boldsymbol{k})$ whose support is denoted by $\mathbb{B}_{\boldsymbol{k}}:=\mathbb{B}+\boldsymbol{k}$. It is not hard to see that there exists a constant $C_{s}$ such that $\left(1+\|\boldsymbol{\xi}\|^{2}\right)^{-s / 2} \leq C_{s}(1+\|\boldsymbol{k}\|)^{-s}$, for all $\boldsymbol{\xi} \in \mathbb{B}_{\boldsymbol{k}}$. Also, let us put $\varphi_{\boldsymbol{k}, s}:=$ $\left(1+\|\cdot\|^{2}\right)^{-s / 2} \varphi_{\boldsymbol{k}}$. With these notations, we now perform the manipulation

$$
\begin{aligned}
\sum_{\boldsymbol{k} \in \mathbb{Z}^{d}}|\langle\hat{f}(\cdot+\boldsymbol{k}), \varphi\rangle| & =\sum_{\boldsymbol{k} \in \mathbb{Z}^{d}}\left|\left\langle\hat{f}, \varphi_{\boldsymbol{k}}\right\rangle\right| \\
& =\sum_{\boldsymbol{k} \in \mathbb{Z}^{d}}\left|\left\langle\left(1+\|\cdot\|^{2}\right)^{\frac{s}{2}} \hat{f},\left(1+\|\cdot\|^{2}\right)^{-\frac{s}{2}} \varphi_{\boldsymbol{k}}\right\rangle\right| \\
& =\sum_{\boldsymbol{k} \in \mathbb{Z}^{d}}\left|\left\langle\mathcal{F}^{-1}\left\{\left(1+\|\cdot\|^{2}\right)^{\frac{s}{2}} \hat{f}\right\}, \mathcal{F}\left\{\varphi_{\boldsymbol{k}, s}\right\}\right\rangle\right|
\end{aligned}
$$




$$
\begin{aligned}
& =\sum_{\boldsymbol{k} \in \mathbb{Z}^{d}}\left|\left\langle J^{s} f, \mathcal{F}\left\{\varphi_{\boldsymbol{k}, s}\right\}\right\rangle\right| \\
& =\sum_{\boldsymbol{k} \in \mathbb{Z}^{d}} \mid\left\langle\mathcal{F}\left\{\left(J^{s} f\right) w^{-1}\right\},\left.\mathcal{F}^{-1}\left\{w \mathcal{F}\left\{\varphi_{\boldsymbol{k}, s}\right\}\right\}\right|_{\mathbb{R}^{d}}\right|,
\end{aligned}
$$

where (31) and (32) are both due to Parseval's relation. As $w(x)=\left(1+\|\boldsymbol{x}\|^{2}\right)^{n}=$ $\sum_{|\ell| \leq n} \frac{n !}{\ell !} x^{2 \ell}$, it follows from the differentiation property of the Fourier transform mentioned in (6) that

$$
\mathcal{F}^{-1}\left\{w \mathcal{F}\left\{\varphi_{\boldsymbol{k}, s}\right\}\right\}(\boldsymbol{\xi})=\sum_{|\ell| \leq n} \frac{n !}{\ell !} \frac{1}{(2 \pi \mathrm{j})^{2|\ell|}} \partial^{2 \ell} \varphi_{\boldsymbol{k}, s}(\boldsymbol{\xi})
$$

This implies that the support of $\mathcal{F}^{-1}\left\{w \mathcal{F}\left\{\varphi_{\boldsymbol{k}, s}\right\}\right\}$ is the same as that of $\varphi_{\boldsymbol{k}, s}$. Thus, we can replace the integration domain $\mathbb{R}^{d}$ in the RHS of (32) by $\mathbb{B}_{\boldsymbol{k}}$. On the other hand, by Leibniz' rule, for every multi-index $\ell$, we have that

$$
\begin{aligned}
\partial^{\ell} \varphi_{\boldsymbol{k}, s}(\boldsymbol{\xi}) & =\partial^{\ell}\left(\left(1+\|\boldsymbol{\xi}\|^{2}\right)^{-s / 2} \varphi_{\boldsymbol{k}}(\boldsymbol{\xi})\right) \\
& =\sum_{\boldsymbol{m}+\boldsymbol{n}=\boldsymbol{\ell}} \frac{\boldsymbol{\ell} !}{\boldsymbol{m} ! \boldsymbol{n} !} \partial^{\boldsymbol{m}}\left(1+\|\boldsymbol{\xi}\|^{2}\right)^{-s / 2} \partial^{\boldsymbol{n}} \varphi_{\boldsymbol{k}}(\boldsymbol{\xi})
\end{aligned}
$$

From this and Lemma 1, we obtain

$$
\left|\partial^{\ell} \varphi_{\boldsymbol{k}, s}(\boldsymbol{\xi})\right| \leq C_{\boldsymbol{\ell}, s}\left(1+\|\boldsymbol{\xi}\|^{2}\right)^{-s / 2} \sum_{\boldsymbol{m}+\boldsymbol{n}=\boldsymbol{\ell}} \frac{\boldsymbol{\ell} !}{\boldsymbol{m} ! \boldsymbol{n} !}\left|\partial^{\boldsymbol{n}} \varphi_{\boldsymbol{k}}(\boldsymbol{\xi})\right|, \quad \forall \boldsymbol{\ell} \in \mathbb{N}^{d} .
$$

Plugging this inequality into (33) yields

$$
\left|\mathcal{F}^{-1}\left\{w \mathcal{F}\left\{\varphi_{\boldsymbol{k}, s}\right\}\right\}(\boldsymbol{\xi})\right| \leq C_{n, s}\left(1+\|\boldsymbol{\xi}\|^{2}\right)^{-s / 2} \sum_{|\ell| \leq 2 n}\left|\partial^{\ell} \varphi_{\boldsymbol{k}}(\boldsymbol{\xi})\right|, \quad \forall \boldsymbol{\xi} \in \mathbb{R}^{d}
$$

We continue to estimate the RHS of (32) as

$$
\begin{aligned}
& \sum_{\boldsymbol{k} \in \mathbb{Z}^{d}}\left|\langle\hat{f}(\cdot+\boldsymbol{k}), \varphi\rangle=\sum_{\boldsymbol{k} \in \mathbb{Z}^{d}}\right|\left\langle\mathcal{F}\left\{\left(J^{s} f\right) w^{-1}\right\}, \mathcal{F}^{-1}\left\{w \mathcal{F}\left\{\varphi_{\boldsymbol{k}, s}\right\}\right\}\right\rangle_{\mathbb{B}_{\boldsymbol{k}}} \mid \\
& \leq \sum_{\boldsymbol{k} \in \mathbb{Z}^{d}}\left\|\mathcal{F}\left\{\left(J^{s} f\right) w^{-1}\right\}\right\|_{L_{2}\left(\mathbb{B}_{\boldsymbol{k}}\right)}\left\|\mathcal{F}^{-1}\left\{w \mathcal{F}\left\{\varphi_{\boldsymbol{k}, s}\right\}\right\}\right\|_{L_{2}\left(\mathbb{B}_{\boldsymbol{k}}\right)} \\
& \leq \sum_{\boldsymbol{k} \in \mathbb{Z}^{d}}\left\|\mathcal{F}\left\{\left(J^{s} f\right) w^{-1}\right\}\right\|_{L_{2}\left(\mathbb{B}_{\boldsymbol{k}}\right)} C_{n, s}(1+\|\boldsymbol{k}\|)^{-s}\left\|\sum_{|\boldsymbol{\ell}| \leq 2 n}\left|\partial^{\ell} \varphi_{\boldsymbol{k}}\right|\right\|_{L_{2}\left(\mathbb{B}_{\boldsymbol{k}}\right)} \\
& \leq C_{n, s} \sum_{|\ell| \leq 2 n}\left\|\partial^{\ell} \varphi\right\|_{L_{2}(\mathbb{B})} \sum_{\boldsymbol{k} \in \mathbb{Z}^{d}}\left\|\mathcal{F}\left\{\left(J^{s} f\right) w^{-1}\right\}\right\|_{L_{2}\left(\mathbb{B}_{\boldsymbol{k}}\right)}(1+\|\boldsymbol{k}\|)^{-s}
\end{aligned}
$$




$$
\begin{aligned}
\leq & C_{n, s} \sum_{|\ell| \leq 2 n}\left\|\partial^{\ell} \varphi\right\|_{L_{2}\left(\mathbb{R}^{d}\right)}\left(\sum_{\boldsymbol{k} \in \mathbb{Z}^{d}}(1+\|\boldsymbol{k}\|)^{-2 s}\right)^{1 / 2} \\
& \times\left(\sum_{k \in \mathbb{Z}^{d}}\left\|\mathcal{F}\left\{\left(J^{s} f\right) w^{-1}\right\}\right\|_{L_{2}\left(\mathbb{B}_{\boldsymbol{k}}\right)}^{2}\right)^{1 / 2} \\
\leq & C_{n, s} \sum_{|\ell| \leq 2 n}\left\|\partial^{\ell} \varphi\right\|_{L_{2}\left(\mathbb{R}^{d}\right)}\left(\sum_{k \in \mathbb{Z}^{d}}\left\|\mathcal{F}\left\{\left(J^{s} f\right) w^{-1}\right\}\right\|_{L_{2}\left(\mathbb{B}_{\boldsymbol{k}}\right)}^{2}\right)^{1 / 2} \\
\leq & C_{n, s} \sum_{|\ell| \leq 2 n}\left\|\partial^{\ell} \varphi\right\|_{L_{2}\left(\mathbb{R}^{d}\right)}\left\|\mathcal{F}\left\{\left(J^{s} f\right) w^{-1}\right\}\right\|_{L_{2}\left(\mathbb{R}^{d}\right)} \\
= & C_{n, s}\left\|\left(J^{s} f\right) w^{-1}\right\|_{L_{2}\left(\mathbb{R}^{d}\right)} \sum_{|\ell| \leq 2 n}\left\|\partial^{\ell} \varphi\right\|_{L_{2}\left(\mathbb{R}^{d}\right)} \\
= & C_{n, s}\|f\|_{L_{2,-2 n}^{s}\left(\mathbb{R}^{d}\right)}\|\varphi\|_{L_{2}^{2 n}\left(\mathbb{R}^{d}\right)}
\end{aligned}
$$

where (35) is a consequence of the Cauchy-Schwarz inequality; (36) follows from (34) and from the bound $\left(1+\|\boldsymbol{\xi}\|^{2}\right)^{-s / 2} \leq C_{s}(1+\|\boldsymbol{k}\|)^{-s}$ over $\mathbb{B}_{\boldsymbol{k}} ;$; (37) was obtained by a change of variable and by Minkowski's inequality;(38) follows from the CauchySchwarz inequality for sequences; (39) is because $2 s>d$; (40) is due to the compactness of the support $\mathbb{B}$; (41) follows from Parseval's relation; and, finally, the desired bound (42) was obtained by the definitions of Sobolev norms in (14) and (17) and by the equivalence between $\|\cdot\|_{H_{2}^{2 n}}\left(\mathbb{R}^{d}\right)$ and $\|\cdot\|_{L_{2}^{2 n}}\left(\mathbb{R}^{d}\right)$.

\section{References}

1. Adams, R., Aronszajn, N., Smith, K.T.: Theory of Bessel potentials. Part II. Ann. Inst. Fourier 17, $1-135$ (1967)

2. Adams, R.A.: Sobolev Spaces. Academic Press, Boston (1975)

3. Aronszajn, N., Smith, K.T.: Theory of Bessel potentials. Part I. Ann. Inst. Fourier 11, 385-475 (1961)

4. Benedetto, J.J., Zimmermann, G.: Sampling multipliers and the Poisson summation formula. J. Fourier Anal. Appl. 3(5), 505-523 (1997)

5. Bergh, J., Löfström, J.: Interpolation Spaces. Springer, Beilin (1976)

6. Bracewell, R.N.: The Fourier Transform and Its Applications. McGraw-Hill, New York (1999)

7. Campbell, L.L.: Sampling theorem for the Fourier transform of a distribution with bounded support. SIAM J. Appl. Math. 16(3), 626-636 (1968)

8. Fischer, J.V.: On the duality of discrete and periodic functions. Mathematics 3(2), 299-318 (2015)

9. Grafakos, L.: Modern Fourier Analysis, 2nd edn. Springer, New York (2008)

10. Grafakos, L.: Classical Fourier Analysis, 2nd edn. Springer, New York (2008)

11. Gröchenig, K.: An uncertainty principle related to the Poisson summation formula. Studia Math. 121(1), 87-104 (1996)

12. Hoskins, R.F., Sousa Pinto, J.: Sampling expansions for functions band-limited in the distributional sense. SIAM J. Appl. Math. 44, 605-610 (1984)

13. Hoskins, R.F., Sousa Pinto, J.: Distributions, Ultra-distributions and Other Generalised Functions. Ellis Horwood, New York (1994)

14. Kahane, J.-P., Lemarié-Rieusset, P.G.: Remarques sur la formule sommatoire de Poisson. Studia Math. 109(3), 303-316 (1994) 
15. Katznelson, Y.: An Introduction to Harmonic Analysis, 3rd edn. Cambridge University Press, Cambridge (2004)

16. Lee, A.J.: Characterization of bandlimited functions and processes. Inform. Contr. 31, 258-271 (1976)

17. Leoni, G.: A First Course in Sobolev Spaces. American Mathematical Society, Providence (2009)

18. Nguyen, H.Q., Unser, M.: A sampling theory for non-decaying signals. Appl. Comput. Harmon. Anal. Available: doi:10.1016/j.acha.2015.10.006 (2015)

19. Nguyen, H.Q., Unser, M.: Generalized Poisson summation formula for tempered distributions. In Proceeding of the 11th International Conferene Sampling Theory and Applications (SampTA'15), May 25-29, pp. 1-5. (2015)

20. Oppenheim, A.V., Willsky, A.S.: Signals and Systems, 2nd edn. Prentice-Hall, Upper Saddle River (1997)

21. Papoulis, A.: The Fourier Integral and Its Applications. McGraw-Hill, New York (1962)

22. Pfaffelhuber, E.: Sampling series for band-limited generalized functions. IEEE Trans. Inform. Theory 17(6), 650-654 (1971)

23. Rudin, W.: Functional Analysis, 2nd edn. McGraw-Hill, New York (1991)

24. Schwartz, L.: Théorie des Distributions. Hermann, Paris (1966)

25. Shannon, C.: Communication in the presence of noise. Proc. IRE 37(1), 10-21 (1949)

26. Stein, E.: Singular Integrals and Differentiability Properties of Functions. Princeton University Press, Princeton (1970)

27. Stein, E., Weiss, G.: Introduction to Fourier Analysis on Euclidean Spaces. Princeton University Press, Princeton (1971)

28. Tao, T.: Sobolev spaces. Available: https://terrytao.wordpress.com/2009/04/30/245c-notes-4-sob olev-spaces/ (2009)

29. Terras, A.: Harmonic Analysis on Symmetric Spaces and Applications. Springer, New York (1985)

30. Unser, M., Blu, T.: Fractional splines and wavelets. SIAM Rev. 42(1), $43-67$ (2000)

31. Unser, M., Tafti, P.D.: An Introduction to Sparse Stochastic Processes. Cambridge University Press, Cambridge (2014)

32. Walter, G.G.: Sampling bandlimited functions of polynomial growth. SIAM J. Math. Anal. 19(5), $1198-1203$ (1988)

33. Walter, G.G.: Nonuniform sampling of bandlimited functions of polynomial growth. SIAM J. Math. Anal. 23(4), 995-1003 (1992)

34. Yamamoto, Y.: From Vector Spaces to Function Spaces: Introduction to Functional Analysis with Applications. Society for Industrial and Applied Mathematics (SIAM), Philadelphia (2012)

35. Zayed, A.I., García, A.G.: Nonuniform sampling of bandlimited signals with polynomial growth on the real axis. IEEE Trans. Inform. Theory 43(5), 1717-1721 (1997)

36. Zygmund, A.: Trigonometric Series, 3rd edn. Cambridge University Press, Cambridge (2003) 